\title{
4. Does the 2008 NATSISS underestimate the prevalence of high risk Indigenous drinking?
}

\author{
Tanya Chikritzhs and Wenbin Liang
}

The 2008 National Aboriginal and Torres Strait Islander Social Survey (NATSISS) estimated that some 9 per cent of Indigenous males and 3.7 per cent of Indigenous females (6.3\% for males and females combined) consumed alcohol at levels which placed them at high risk of chronic harm (Australian Bureau of Statistics (ABS) 2010a). This was similar to that estimated by the 2002 NATSISS of 5.6 per cent for males and females combined (ABS 2004). ${ }^{1}$ Levels of alcohol use which place the drinker at high risk for chronic harm were defined by the 2001 National Health and Medical Research Council (NHRMC) guidelines as consuming, on average, more than six standard drinks a day for males and more than four for a female. The levels themselves were established based on studies of the relationship between alcohol exposure and alcohol-attributable chronic harms (NHRMC 2001). Chronic alcohol-attributable harms include conditions which result from long term exposure to consistent heavy alcohol use including, in particular, alcoholic liver cirrhosis, alcohol dependence (a chronic condition by definition) and a range of less prevalent conditions (e.g. alcoholic pancreatitis, some cancers for which alcohol is partially attributable). ${ }^{2}$

Chikritzhs and Brady (2006a, 2007) argued that the 2002 NATSISS substantially underestimated actual levels of chronic risky/high risk alcohol use in the Indigenous population. They compared the results from the 2002 NATSISS to other national surveys of Indigenous alcohol use and general population surveys and found the outcomes from the 2002 NATSISS to be implausibly low. The 2002 NATSISS estimated that about 15.2 per cent of Indigenous Australians (aged 15 years and over) drank at levels that placed them either at risk or high-risk ${ }^{3}$ for chronic alcohol-related harm (ABS 2004) - the 2008 estimate was similar at 17.2 per cent (ABS 2010a). ${ }^{4}$ A particularly stark comparison was drawn with the 1994 National Drug Strategy Household Survey: Urban Aboriginal and Torres Strait

\footnotetext{
1 For comparison purposes, the 2002 and 2008 surveys are methodologically similar (ABS 2010b).

2 It should be noted that the 2001 NHMRC guidelines have been superseded by the 2009 NHMRC guidelines which recommend no more than 2 standard drinks per day on average and 4 standard drinks for a single occasion for both males and females.

3 'Risky use' is defined as more than 4 standard drinks per day for males and more than 2 standard drinks per day for females.

4 The 2002 NATSISS and 2008 NATSISS also estimated similar proportions of Indigenous people as drinking at risk/high risk levels for acute harm in the 2 weeks before the survey ( $35 \%$ and $37 \%$ respectively).
} 
Islander Peoples Supplement ${ }^{5}$ (Commonwealth Department of Human Services and Health (CDHSH) 1996; referred to below as the 1994 urban Indigenous survey) which estimated that over 50 per cent of regular Indigenous drinkers consumed alcohol at levels which placed them at risk or high risk of harm. The 1994 urban Indigenous survey also compared prevalence estimates of high risk consumption among regular Indigenous drinkers to regular non-Indigenous drinkers (from a 1993 urban general population survey), the ratio for which was about 5.4 (based on all respondents). ${ }^{6}$ In other words, for every one high-risk regular drinker among the non-Indigenous population there were at least five Indigenous drinkers consuming alcohol at high risk levels (CDHSH 1996).

One of the main applications for surveys of alcohol and other drug use is the identification of the range and magnitude of use within a population and how it may differ among sub-populations. It is not unreasonable to expect that large national surveys of substance use can be relied upon as fairly accurate indicators of exposure and, by extension, likely levels of harms occurring due to problematic use. The reality, however, is that all alcohol surveys are prone to underestimating actual levels of use. Most surveys typically account for only 40 per cent to 60 per cent of known alcohol sales in a community (Knibbe and Bloomfield 2001), and Stockwell et al. (2008) have demonstrated that on average, respondents to the 2004 National Drug Strategy Household Survey (NDSHS) underestimated their consumption by about 28 per cent - largely due to recall bias. The factors which influence under-reporting in surveys may vary widely depending on the methods used and the population surveyed. Chikritzhs and Brady (2006a) highlighted three potential sources of underestimation in the 2002 NATSISS:

- the use of face-to-face interviews and lack of confidentiality for respondents

- problematic data collection methods, and

- exclusion of residents living in non-private dwellings. In relation to the alcohol component, little has changed for the 2008 NATSISS and these potential sources of error remain salient.

Given the limitations of surveys, triangulation with other data sources on harms and consumption is a useful means of verifying their veracity and highlighting discrepancies. Chikritzhs and Brady (2006a, 2006b) made a preliminary attempt to compare the risky/high risk prevalence estimates from the 2002 NATSISS to independent sources of information on alcohol-related deaths and hospitalisations comparing Indigenous and non-Indigenous people to the

5 Urban was defined as a minimum population of 1000.

6 Among all respondents, i.e. not restricted to drinkers. The ratio among only drinkers was about 6.6 (derived from CDHSH 1996: 30, Table 21; note that the relevant Table did not provide sex-specific proportions). This ratio is based on amounts usually consumed, harmful defined as more that 4 standard drinks for females and more than 6 standard drinks for males among current regular drinkers. 
general population. They noted for instance that the Steering Committee for the Review of Government Service Provision (2005) cited 2002-03 Indigenous population rates for alcoholic liver disease and alcohol dependence which were 5 and 2.4 times higher respectively than for the non-Indigenous population. From 1990 to 1997, compared to their non-Indigenous counterparts, the Indigenous death rate from all wholly alcohol attributable conditions in Western Australia, South Australia and the Northern Territory combined was about 8 times higher for males and 16 times higher for females (Chikritzhs et al. 2000). Yet, the 2002 NATSISS indicated levels of chronic risky/high risk consumption among Indigenous people which were less than twice that for non-Indigenous people. More recently, despite the known and extreme disparity in alcohol-attributable death rates between Indigenous and non-Indigenous Australians (i.e. up to $800 \%$ greater for Indigenous people), a comparison of the 2008 NATSISS to the 2007 NDSHS of the general population (Australian Institute of Health and Welfare (AIHW) 2008) suggests that the discrepancy in the prevalence of high risk drinking between the two groups is only 67 per cent (i.e. $17.2 \%$ vs $10.3 \%$ ). ${ }^{7}$ As Chikritzhs and Brady (2006a) point out, the discord between rate of death from disease caused specifically by alcohol and apparent rate of exposure among the Indigenous compared to the general population is not easily reconciled.

In this chaper, we extend the triangulation approach taken by Chikritzhs and Brady (2006a, 2006b) to highlight the implausibility of the 2008 NATSISS. Alcoholic liver cirrhosis and alcohol dependence are two sentinel conditions well established as arising from long term (chronic) exposure to heavy alcohol use (NHMRC 2001; World Health Organization (WHO) 2000). Among death statistics, these two conditions are also relatively common causes of death compared to other wholly alcohol-attributable conditions (e.g. alcoholic cardiomyopathy, alcoholic gastritis, alcoholic pancreatitis). Our approach relies on comparing sex-specific Indigenous and non-Indigenous death rates for alcoholic liver cirrhosis and alcohol dependence while taking into account the magnitude of the populations at high risk indicated by the 2008 NATSISS and the 2007 NDSHS (i.e. the 'exposed' population). We begin this investigation as if the veracity of the 2007 NDSHS were not in question (i.e. not underestimated) and assuming that it gives a reasonable approximation of the true prevalence of high risk drinking for chronic harm in the general population. From this, we estimate the potential magnitude of underestimation of the actual proportion of the Indigenous high risk drinking population by the 2008 NATSISS.

7 Both the NDSHS and the NATSISS ask respondents to recall usual alcohol use in the past 12 months. 


\section{Method}

\section{Surveys}

The estimated age and sex specific prevalence of high risk alcohol consumption for chronic harms for the Indigenous population was obtained from the 2008 NATSISS (ABS 2010a). The estimated age and sex specific prevalence of high risk alcohol consumption for chronic harms for the general population was obtained from the 2007 NDSHS report on first results (AIHW 2008).

The 2008 NATSISS included 7342 Indigenous respondents aged 15-64 years (ABS 2009) and the 2007 NDSHS included 23356 respondents from the general population aged $14+$ years (AIHW 2008).

Both the 2008 NATSISS and the 2007 NDSHS based their drinking prevalence estimates on levels defined by the 2001 NHMRC drinking guidelines. The 2001 NHMRC guidelines defined alcohol consumption which places the drinker at high risk for chronic harm (long-term harm) for males at seven or more standard drinks per day on average or 43 or more per week, and for females at five or more standard drinks per day on average or 29 or more per week. A standard Australian drink is considered to be 10 grams or 12.5 millilitres (mls) of pure alcohol (NHMRC 2001).

Both surveys asked respondents to recall and report their usual consumption over the past 12 months. In the NDSHS, respondents were asked to complete a graduated quantity frequency table and report their consumption in units of standard drinks with the aid of a show card. The NATSISS did not require respondents to report their consumption as numbers of standard drinks but asked respondents to report their usual consumption in terms of typical container sizes which were later converted into millilitres of pure alcohol by the ABS.

Respondents to the NDSHS self-complete their answers to substance use questions without the overview of an interviewer. Their responses (which do not include name or address details) were sealed in an envelope and returned to the field worker or mailed back to the collection agency. For the 2008 NATSISS, data were collected using face-to-face interviews and respondent answers were verbally related to, and recorded on a computer (for non-remote respondents) by the interviewer. Moreover, although the interviewer was instructed to suggest to respondents that the interview take place in a private, other household members may have been present. 
Notably, the NATSISS procedure was somewhat different for questioning related to 'substance use' which included the misuse of prescription drugs and/or the use of illicit drugs such that:

Due to the potentially sensitive nature of the questions, responses to these questions were voluntary. In non-remote areas, people answered questions through a voluntary self-completion substance use form. In remote areas, people were personally interviewed (ABS 2010b: no page numbers).

The 2007 NDSHS included a small number of Indigenous respondents (less than $2 \%$ ) who were apparently included in the general population prevalence estimates. Given the likely negligible impact of a small number of Indigenous responses on the overall drinking prevalence of the entire sample it was deemed appropriate for the purposes of this analysis to consider the 2007 NDSHS sample representative of the non-Indigenous national population.

\section{Death data}

Unit records of Australian deaths reported from 2000 to 2006 were obtained from the ABS including primary cause of death (ICD-10 coded), age at death, sex, and year of death. Primary cause of death was used to identify deaths from alcoholic liver cirrhosis and alcohol dependence. Cause of death is usually determined by coronial officers. The reliability of Indigenous status flags for death records is high (Chikritzhs et al. 2004).

\section{Estimated residential population}

Estimated residential population (ERP) for the national non-Indigenous and Indigenous populations aged by five-year age cohorts $(0-4,5-9,10-14,15-19 \ldots$ $65+$ ) and sex were obtained from the 2006 ABS Census (ABS 2007).

\section{Analysis}

Random effects panel Poisson regression modelling was used to estimate the sex-specific incidence risk ratio of alcohol caused death by Indigenous status. Poisson regression is preferable where counts of events are modelled and is suitable for small numbers of observations. Counts of deaths from 2000 to 2006 caused by alcoholic liver cirrhosis or alcohol dependence (for ages 15+) formed the dependent variable. Panels were determined by combinations of calendar years and age groups (15-19, 20-24, 25-29...65+). 
The exposure variable was the number of people estimated to be at high risk for chronic alcohol-related harm in the Indigenous and non-Indigenous populations based on the population prevalence estimates for high risk chronic drinking derived from the 2008 NATSISS and the 2007 NDSHS. According to the surveys, the age- and gender-specific population supposed to have consumed alcohol at high risk levels was calculated by multiplying the prevalence of high risk drinking and population estimates from the census for each age-gender strata by Indigenous status. For example, the 2008 NATSISS estimated that 7.6 per cent of Indigenous males aged 25-29 years consumed alcohol at a high risk level for chronic harm and in 2006 the Indigenous male population in that age group was estimated to be 14 932, thus, the number of 25-30 year old Indigenous males estimated to be at high risk on the basis of the NATSISS was 1135 (14 $932^{*} 0.076$ ). A similar procedure was followed to estimate the exposure variable for the non-Indigenous population, based on the age- and gender-specific population prevalence estimates reported by the 2007 NDSHS.

\section{Results}

\section{Drinking prevalence estimates}

The 2008 NATSISS and the 2007 NDSHS provided summaries of the prevalence of high risk drinking for chronic harm. Both were based on the 2001 NHMRC drinking guidelines for identifying high risk drinking for chronic harm and both asked respondents to report their usual consumption in the past 12 months. The summaries of the relevant survey results as they appear in AIHW (2008) and ABS (2010a) reports have been compiled in Table 4.1.

Table 4.1 High risk alcohol consumption, estimated Indigenous and nonIndigenous populations, Australia, 2007-08

\begin{tabular}{|c|c|c|c|c|c|c|c|}
\hline \multicolumn{4}{c}{ Indigenous $^{\mathrm{b}}$} \\
& Male & \multicolumn{1}{c|}{ Female $^{2}$ Total } & \multicolumn{4}{c|}{ Male } & \multicolumn{2}{c|}{ Female } & \multicolumn{1}{c|}{ Total } \\
\hline $15-24$ & 7.9 & 3.6 & 5.7 & $14-19$ & 2.6 & 3.9 & 3.2 \\
\hline $25-34$ & 7.6 & 5.1 & 6.3 & $20-29$ & 6.2 & 5.4 & 5.8 \\
\hline $35-44$ & 11.2 & 3.9 & 7.2 & $30-39$ & 3.7 & 3.0 & 3.3 \\
\hline $45-54$ & 12.9 & 3.0 & 7.7 & $40-49$ & 3.5 & 2.6 & 3.1 \\
\hline $55+$ & 6.9 & 2.6 & 4.5 & $50-59$ & 5.1 & 2.7 & 3.9 \\
\hline & & & & $60+$ & 2.5 & 0.8 & 1.6 \\
\hline Total & 9.0 & 3.7 & 6.3 & Total & 3.9 & 2.8 & 3.4 \\
\hline
\end{tabular}

a. The age groups provided in the summary reports of the two surveys were not identical.

b. From 2008 NATSISS. 
As summarised in Table 4.2, the all-ages estimate of male Indigenous high risk drinking for chronic harm from the 2008 NATSISS was more than twice the estimate for non-Indigenous males made by the 2007 NDSHS. The Indigenous and non-Indigenous female estimates were notably similar. Overall, on the basis of the 2008 NATSISS, Indigenous drinkers at risk for chronic harms appear to outnumber non-Indigenous drinkers estimated by the 2007 NDSHS by 1.85 to 1 .

Table 4.2 High risk alcohol consumption, all ages, Indigenous and nonIndigenous populations, Australia, 2007-08

\begin{tabular}{|c|c|c|c|}
\hline & Indigenous $^{a}$ & Non-Indigenous ${ }^{b}$ & Ratio \\
\hline Male & 9.0 & 3.9 & 2.31 \\
\hline Female & 3.7 & 2.8 & 1.32 \\
\hline Rate ratio & 2.4 & 1.4 & \\
\hline Total & 6.3 & 3.4 & 1.85 \\
\hline
\end{tabular}
a. From 2008 NATSISS
b. From 2007 NDSHS.

\section{Death rates and overall high risk drinking prevalence}

As shown in Table 4.3, from 2000 to 2006 there were 5065 non-Indigenous deaths and 425 Indigenous deaths (15+ years) attributed to either alcoholic liver cirrhosis or alcohol dependence. Adjusted for residential population, over the seven year period, this represented a population death rate of about 2.0 per 10000 for the Indigenous population and 0.44 per 10000 for the non-Indigenous population. Thus, for every 1 non-Indigenous death about 4.5 Indigenous people died from causes known to be attributable to chronic heavy alcohol use. The rate ratio for Indigenous versus non-Indigenous was particularly high for females. Although death records indicate that Indigenous females suffer a death rate from alcoholic liver cirrhosis and alcohol dependence almost eight times greater than their non-Indigenous counterparts, a comparison indicating the potential populations at risk yields only a marginal ratio of 1.30 .

Table 4.3 Death rates from alcoholic liver cirrhosis and alcohol dependence, Indigenous and non-Indigenous population, Australia, 2000-06

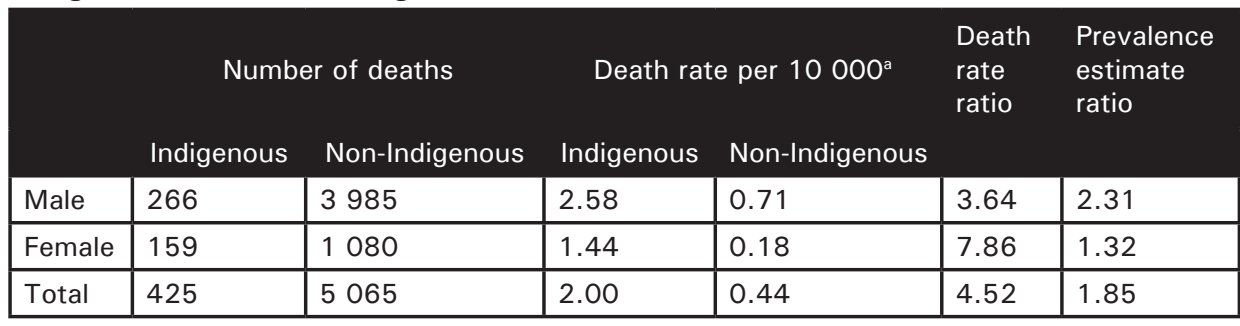




\section{Regression analysis}

Poisson regression modelling indicated that given high risk populations estimated by the NATSISS and the NDSHS, the death rate from either liver cirrhosis or alcohol dependence among high risk drinkers was about 2.2 (95\% CI $1.96,2.53)$ times higher for Indigenous compared to non-Indigenous males and about $7.2(95 \%$ CI $6.14,8.57)$ times higher among Indigenous females compared to non-Indigenous females (see Table 4.4).

For Indigenous deaths only, the incidence rate ratio (IRR) for females to males was 1.54 indicating that, based on exposure indicated by high risk drinking from the 2008 NATSISS, the death rate was greater for females. In the reverse, for the non-Indigenous population, the IRR was 0.50 indicating females were less likely to die from alcoholic liver cirrhosis and alcohol dependence.

Table 4.4 Deaths from alcoholic liver cirrhosis and alcohol dependence, by Indigenous status and gender (Poisson regression modelling), Australia, 2000-06

\begin{tabular}{|l|c|c|c|c|}
\hline \multicolumn{7}{|c|}{ IRR } & $\begin{array}{c}95 \% \text { Confidence interval } \\
\text { Lower value } \\
\text { Upper }\end{array}$ \\
\hline Indigenous status & 1.96 & 2.52 & 0.000 \\
\hline Males: Indigenous (1) vs non Indigenous (0) & 2.22 & 6.14 & 8.57 & 0.000 \\
\hline Females: Indigenous (1) vs non Indigenous (0) & 7.25 & 6.47 & 0.54 & 0.000 \\
\hline Sex & 0.50 & 0.47 & 1.88 & 0.000 \\
\hline Non-Indigenous: females (1) vs males (0) & 1.54 & 1.26 & & \\
\hline Indigenous: females (1) vs males (0) & & &
\end{tabular}

Number in sample $=5$ 490. Death rates for all wholly attributable conditions were also examined and similar outcomes found, see Appendix 4A Table 4A.1.

Source: ABS death unit records 2000-06

Using the Poisson regression results from Table 4.4 it is possible to estimate expected sex-specific ranges for the prevalence of drinking at high risk for chronic harm in the Indigenous population. The estimates below are based on the following assumptions:

- for any population, there is a linear relationship between the number of alcohol attributable deaths from alcoholic liver cirrhosis/alcohol dependence and the size of the population at high risk (i.e. as estimated by prevalence surveys of high risk chronic drinking)

- deaths from alcoholic liver cirrhosis and alcohol dependence arise from, and are representative of, a high risk chronic drinking sub-population

- the reporting of liver cirrhosis/alcohol deaths within sex-groups are not significantly influenced by Indigenous status, and 
- the NDSHS consumption estimates approximate actual drinking levels in the non-Indigenous population (i.e. the NDSHS prevalence estimates are not substantial underestimates).

\section{Indigenous males vs non-Indigenous males}

Poisson regression on numbers of deaths due to alcohol-caused chronic disease indicated that for Indigenous males, the IRR was 2.2 times non-Indigenous deaths, within a range of 1.96 to 2.52 . Since deaths are known, in order to bring the IRR to unity (i.e. the ratio of Indigenous to non-Indigenous $=1$ ), the Indigenous population denominator, which signifies the population exposure to high risk alcohol use (based on prevalence from the 2008 NATSISS would need to increase substantially. This is described in Equation 1 below:

\section{Equation 1}

$\mathrm{D}=$ number of deaths

$\mathrm{HRP}=$ estimated high risk population as a proportion (from surveys)

$\mathrm{M}=$ male

$\mathrm{i}=$ Indigenous population

$\mathrm{g}=$ general population

$\mathrm{IRR}=$ incidence rate ratio

If, $(\mathrm{DMi} / \mathrm{HRPMi}) /(\mathrm{DMg} / \mathrm{HRPMg})=\mathrm{IRR}=2.22$,

Then, to make IRR equal 1 , that is, $\left(\mathrm{DMi} / \mathrm{HRPMi}^{*} \mathrm{X}\right) /(\mathrm{DMg} / \mathrm{HRPMg})=1$,

$\mathrm{X}$ must $=2.22\left(\right.$ i.e. $\left.2.22^{*}(1 / \mathrm{X})=1\right)$

Thus, the actual proportion of Indigenous males drinking at high risk levels for chronic harm would be expected to occur within a range of 1.96 to 2.52 times that of the current estimated prevalence from the 2008 NATSISS (9.0\%), which is between 17.6 per cent and 22.8 per cent.

\section{Indigenous females vs non-Indigenous females}

For females, Poisson regression results indicated between 6.14 and 8.57 times more deaths among the Indigenous compared to the non-Indigenous population. On this basis, and applying Equation 1 above (substituting the variables relevant to females, we would expect the actual proportion of high risk female drinkers in the Indigenous community to range between 22.7 per cent and 31.7 per cent (i.e. $6.14^{*} 3.7$ and $8.57^{*} 3.7$ ). 


\section{Indigenous females vs Indigenous males}

Poisson regression results show substantially different risks for females versus males for the Indigenous (1.54) and non-Indigenous populations (0.5). If it is the case that the female to male risk in the Indigenous population is in actuality similar to that for the non-Indigenous population (i.e. less likely for females) then the resultant IRR for Indigenous females to males must be a spurious outcome of underestimation in the 2008 NATSISS. Assuming that the IRR demonstrated for the non-Indigenous population is accurate, it is possible to estimate the approximate true value of the prevalence ratio for Indigenous females to Indigenous males. That is, if the ratio between the Indigenous female to male IRR (1.54 and non-Indigenous female to male IRR ( 0.50 should equal 1 , then:

\section{$0.50 / 1.54^{*}(X)=1$, where $X$ must $=0.33$}

Thus, for the Indigenous population, in order to achieve a similar female to male risk profile as the non-Indigenous population, the ratio of Indigenous male to Indigenous female high risk populations should be 0.33 times the current NATSISS estimation of 2.4 and about 0.80 . To achieve this, the Indigenous male $(9.0 \%)$ and female $(3.7 \%)$ prevalence estimates from the 2008 NATSISS would need to converge.

\section{Discussion}

The results from this analysis are in keeping with the proposition that the 2008 NATSISS has substantially underestimated the prevalence of high risk alcohol consumption for chronic harms among the national Indigenous population. The current NATSISS prevalence estimates may be underestimated by over 200 per cent for males and 700 per cent for females. Based on alcoholic liver cirrhosis and alcohol dependence deaths from 2000 to 2006, the proportion of the Indigenous population expected to be drinking at high risk levels for chronic harm was estimated to range from 17.6 per cent to 22.8 per cent for males and 22.7 per cent and 31.7 per cent for females. ${ }^{8}$ On this basis, the overall ratio of Indigenous to non-Indigenous high risk drinking prevalence for chronic harm in the population is expected to be about 4.7 to 1 . This is substantially larger than the ratio of 1.85 to 1 indicated by a straight comparison of the high risk drinking prevalence estimates for Indigenous people in the 2008 NATSISS $(6.3 \%)$ to the general population estimates from the 2007 NDSHS (3.4\%). Moreover, the Indigenous to non-Indigenous high risk drinking ratio derived from the analyses

8 It should be noted that the NHMRC guidelines give a lower cut-off for females $(>4)$ compared to males $(>6)$ 
performed in this study ( 4.7 to 1 ) is in relatively close alignment with the ratio of about 5.4 to 1 produced by the 1994 urban Indigenous survey compared to a 1993 urban general population survey.

The original proposition put forward by Chikritzhs and Brady (2006a) - that the 2002 NATSISS substantially underestimated Indigenous alcohol consumption was based on a range of observations. It is difficult to imagine a credible scenario which would cause high risk levels of alcohol use for chronic harm among the national Indigenous population to fall so substantially from the mid 1990s that the actual ratio of Indigenous to non-Indigenous high risk drinking would currently be less than 2 to 1 . It is hypothetically possible that the 1994 Indigenous urban survey overestimated Indigenous consumption (by a large factor) but given that the major sources of error in substance use surveys tend almost exclusively toward under-reporting of actual consumption, the possibility is remote. In addition, national surveys of the general population do not indicate any exceptionally large increases in high risk consumption for chronic harm since the mid 1990s (Clement et al. 2007) which could account for convergence of the Indigenous and non-Indigenous prevalence estimates. It is far more plausible that the NATSISS underestimates Indigenous alcohol consumption substantially.

Why then, might the NATSISS be so prone to underestimating alcohol consumption? Chikritzhs and Brady (2006a, 2006b) identified several potential sources of error including the use of face-to-face interviews and lack of confidentiality for respondents, problematic data collection methods, and exclusion of residents living in non-private dwellings. An important characteristic which was common to both the 1994 urban Indigenous survey and the 2007 NDSHS, but distinctly absent from the NATSISS, was a clear recognition that participants should be afforded a minimum level of privacy when asked to report their personal alcohol use. As Chikritzhs and Brady (2006b: 231) indicated:

It is important at the outset to acknowledge with candour that questioning Aboriginal or Torres Strait Islander people about their use of alcohol and other drugs is always fraught with difficulty, whatever the circumstance.

For instance, throughout the 1994 urban Indigenous survey, although an interviewer was present and initial questioning was conducted face-to-face, sensitive questions about any type of substance use were contained in a confidential sealed section for the respondent's self-completion, and there was no direct questioning from the interviewer (although the interviewer was able to provide assistance when asked). The general population NDSHS also use a self-complete questionnaire which is sealed by the respondent after completion 
(without any personal identifying information) and returned to the survey field worker or via mail in a reply paid envelope. The 2008 NATSISS (and 2002), however, takes an approach which requires the respondent to verbally relate their alcohol use to the interviewer. This differs to the questions relating to prescription drugs and/or the use of illicit drugs which are voluntary and able to be privately self-completed (at least by non-remote respondents).

It is a remarkable contrast that all the national drug strategy surveys, including the 1994 urban Indigenous survey recognise the personal nature of all alcohol and other drug use questions asked, while repeatedly and explicitly making clear its strict protocol for maintaining privacy. Yet, the ABS takes this view only for drugs other than alcohol (and tobacco). It is even more striking a discord when it is considered that for Indigenous people, alcohol use imparts a social burden well beyond that experienced by the general population and 'carries with it a complex political, legislative and racialised past and is the cause of polarised views among Aboriginal practitioners and commentators' (Chikritzhs and Brady 2006a: 278). In this context, it would be naïve to assume that because alcohol is a legally available drug, that its consumption is not a highly loaded and sensitive issue for individuals, families, and communities. It is not certain that lack of privacy is a major cause of underestimation in the NATSISS, but it is certainly worth investigating further, as are the other potential sources of error.

\section{Limitations}

On the basis of the regression results, it was possible to estimate a more probable range for actual high risk alcohol exposure in the Indigenous population. It should be kept in mind, however, that the intention of this study was not to reestimate the prevalence of Indigenous alcohol consumption, but to highlight, via triangulation with death records, the implausibility of the current estimates of chronic levels of consumption derived from the 2008 NATSISS. The ranges given depend heavily on the assumption that the 2007 NDSHS (which forms the basis for comparison) is a reliable and accurate indicator of high risk consumption in the non-Indigenous population. In fact, the 2007 NDSHS, like most other surveys, almost certainly underestimates the proportion of people in the general population who drink at high risk levels for chronic harm (e.g. Stockwell et al. 2007). In which case, the Indigenous prevalence ranges estimated from the regression analyses would also be underestimated.

It is also possible that Indigenous deaths from alcoholic liver cirrhosis and alcohol dependence are over-reported compared to the non-Indigenous population. For instance, it is possible that coroners tend to focus on alcohol-related causes of death more often for Indigenous people and under-report for non-Indigenous people. Alternatively, the detection and treatment of alcoholic liver cirrhosis 
and alcohol dependence among Indigenous people may be inferior compared to their non-Indigenous counterparts, leading to a greater likelihood of death in the former. However, while this may explain a portion of the difference between Indigenous and non-Indigenous death rates, it is unlikely to fully explain the extremely large discrepancies found. In particular, it does not explain why the discrepancy should be so very much larger for females than for males, nor why the Indigenous female to male risk ratio should be 1.5 (based on Indigenous deaths and the 2008 NATSISS Indigenous prevalence estimates), while the reverse relationship is demonstrated for the non-Indigenous population (0.5).

It is also worth noting that the estimates of drinking prevalence examined here relate to high risk levels of alcohol use only. We have not considered levels of use which would be considered risky use for chronic alcohol related harm (that is, 5-6 standard drinks per day on average for males and 3-4 per day on average for females), which presumably are also likely to be underestimated.

\section{Conclusion}

To the extent possible, triangulation with deaths attributable to chronic heavy alcohol use has supported the proposition that the prevalence of high risk drinking detected by the 2008 NATSISS (and the 2002 NATSISS) is underestimated to a substantial extent. The ABS acknowledges that its surveys underestimate actual consumption levels for alcohol and other substances (e.g. ABS 2009). In this, the ABS surveys are not unique: most surveys of alcohol and drug use underestimate consumption, most of the time. Regarding the NATSISS, the concerns which require further consideration are whether the magnitude of the underestimate is so large that it is beyond reasonable and acceptable bounds of error for national population surveys and whether it should be relied upon as in any way an accurate source of information on Indigenous alcohol consumption. 


\section{Appendix 4A: Tables}

Table 4A.1 Deaths from wholly alcohol attributable conditions (Poisson regression modelling), Australia, 2000-06

\begin{tabular}{|c|c|c|c|c|}
\hline & \multirow[t]{2}{*}{ IRR } & \multicolumn{2}{|c|}{$95 \%$ Confidence interval } & \multirow[t]{2}{*}{$\mathrm{P}$ value } \\
\hline & & Lower & Upper & \\
\hline \multicolumn{5}{|l|}{ Indigenous status } \\
\hline Males: Indigenous ( 1 ) vs non Indigenous (0) & 2.53 & 2.28 & 2.80 & 0.000 \\
\hline Females: Indigenous (1) vs non Indigenous (0) & 6.54 & 5.64 & 7.57 & 0.000 \\
\hline \multicolumn{5}{|l|}{ Sex } \\
\hline Non-Indigenous: females (1) vs males (0) & 0.52 & 0.49 & 0.55 & 0.000 \\
\hline Indigenous: females (1) vs males (0) & 1.24 & 1.04 & 1.47 & 0.014 \\
\hline
\end{tabular}

a. Number in sample $=5955$ males, 1738 females. Includes the following conditions: alcoholic psychosis, alcohol abuse, alcohol dependence, alcoholic cardiomyopathy, alcoholic gastritis, alcoholic liver cirrhosis, alcoholic pancreatitis, alcoholic polyneuropathy, alcoholic poisoning, aspiration (vomitus).

Source: ABS death unit records 2000-06

\section{Acknowledgements}

The authors would like to thank Professor Dennis Gray, Dr Steven Skov and Dr Maggie Brady for their helpful comments on earlier drafts.

\section{References}

Australian Bureau of Statistics (ABS) 2004. National Aboriginal and Torres Strait Islander Social Survey 2002, cat. no. 4714.0.55.001, ABS, Canberra.

2007. 2006 Census of Population and Housing Australia, cat. no. 2068.0, ABS, Canberra.

2009. National Aboriginal and Torres Strait Islander Social Survey 2008: Summary, cat. no. 4714.0, ABS, Canberra.

2010a. National Aboriginal and Torres Strait Islander Social Survey (NATSISS 2008, cat. no. 4714.0, ABS, Canberra.

2010b. National Aboriginal and Torres Strait Islander Social Survey: Users' Guide, 2008, cat. no. 4720.0, ABS, Canberra. 
4. Does the 2008 NATSISS underestimate the prevalence of high risk Indigenous drinking?

Australian Institute of Health and Welfare (AIHW) 2008. 2007 National Drug Strategy Household Survey: First Results, Drug Statistics Series No. 20, cat. no. PHE 98, AIHW, Canberra.

Chikritzhs, T. and Brady 2006a. 'Fact or fiction? A critique of the National Aboriginal and Torres Strait Islander Social Survey, 2002', Drug and Alcohol Review, 25: 277-87.

- and - 2006b. 'Substance use in the 2002 NATSISS', in B. Hunter (ed.), Assessing the Evidence on Indigenous Socioeconomic Outcomes: A Focus on the 2002 NATSISS, CAEPR Research Monograph No. 26, ANU E Press, Canberra.

and - 2007. 'Postscript to: Fact or fiction? A critique of the National Aboriginal and Torres Strait Islander Social Survey, 2002', Drug and Alcohol Review, 26: 221-22.

, Gray, D., Stockwell, T., Stearne, A., Pascal, R. and Saggers, S. 2004. Applying National Indicators of Alcohol-Related Harms to Indigenous Australians: A Discussion Paper, National Drug Research Institute, Curtin University of Technology, Perth.

1997, National Alcohol Indicators Technical Report no. 1, National Drug Research Institute, Curtin University of Technology, Perth.

Clement, S., Donath, S., Stockwell, T. and Chikritzhs, T. 2007. Alcohol Consumption in Australia: National Surveys from 1989 to 2004, Technical Report, National Drug Research Institute, Curtin University of Technology, Perth.

Commonwealth Department of Human Services and Health (CDHSH) 1996. National Drug Strategy Household Survey Urban Aboriginal and Torres Strait Islander Peoples Supplement1994, AGPS, Canberra.

Knibbe, R. and Bloomfield, K. 2001. 'Alcohol consumption estimates in surveys in Europe: Comparability and sensitivity for gender differences', Journal of Subtance Abuse, 22: 23-38.

National Health and Medical Research Council (NHMRC) 2001. Australian Alcohol Guidelines: Health Risks and Benefits, NHMRC, Canberra.

2009. Australian Guidelines to Reduce Health Risks from Drinking Alcohol, NHMRC, Canberra.

Steering Committee for the Review of Government Service Provision 2005. Overcoming Indigenous Disadvantage: Key Indicators 2005, Overview, Productivity Commission, Canberra. 
Stockwell, T., Jinhui, Z., Chikritzhs, T. and Greenfield, T. 2008. 'What did you drink yesterday? Public health relevance of a recent recall method used in the 2004 Australian National Drug Strategy Household Survey', Addiction, 103: 919-28.

World Health Organization (WHO) 2000. International Guide for Monitoring Alcohol Consumption and Alcohol Related Harm, T. Stockwell and T. Chikritzhs (eds), WHO, Geneva, viewed 27 September 2011, available at <http://whqlibdoc.who.int/hq/2000/who_msd_msb_00.4.pdf> 